\title{
Generation and characterization of a monoclonal antibody against duck Tembusu virus envelope protein
}

\author{
K. Han, D. Zhao, Y. Liu, Q. Liu, X. Huang, J. Yang, K. Bi, T. Xu, Y. Li \\ Institute of Veterinary Medicine, Jiangsu Academy of Agricultural Sciences, Key Laboratory of Veterinary \\ Biological Engineering and Technology, Ministry of Agriculture, \\ National Center for Engineering Research of Veterinary Bio-products, Nanjing, Jiangsu Province, PR China; \\ Jiangsu Co-innovation Center for Prevention and Control of Important Animal Infectious Diseases and Zoonoses, \\ Yangzhou, 225009
}

\begin{abstract}
Duck Tembusu virus (DTMUV) is a newly emerging pathogenic flavivirus that has caused massive economic losses to the duck industry in China. Envelope (E) protein of DTMUV is an important structural protein, which is able to induce protective immune response in target animals and can be used as specific serological diagnosis tool. In this study, a novel monoclonal antibody, designated mAb 3E9, was generated against DTMUV E protein. It is positive in indirect ELISA against both His-E protein and the purified whole viral antigen. Also, this mAb showed positive reaction with DTMUV in Western blot and indirect immunofluorescence assay, and the isotype was IgG1. End-point neutralizing assay performed in BHK-21 cells revealed that the neutralization titer of 3E9 against DTMUV JS804 strain reached 1:50. Furthermore, functional studies revealed that 3E9 blocks infection of DTMUV at a step on viral attachment. The anti-E mAbs produced in the present work may be valuable in developing an antigen-capture ELISA test for antigen detection or a competitive ELISA test for antibody detection or therapeutic medicine for DTMUV in poultry.
\end{abstract}

Key words: duck Tembusu virus, envelope protein, monoclonal antibody

\section{Introduction}

In April 2010, a severe duck disease has emerged throughout the main duck-producing regions of eastern China. The infected layer ducks developed a significant reduction in egg production ranging from $20 \%$ to $60 \%$, even up to $90 \%$, with mortality rate varying from 5\% to 30\% (Cao et al. 2011, Su et al. 2011). The diseased ducks developed some nervous system disorders including unsteady standing, falling and quivering ( $\mathrm{Ti}$ et al. 2015). In addition to ducks, this disease has affected geese, chickens, and sparrows (Sun et al. 2014). The emerging disease was designated duck hemorrhagic ovaritis (DHO) firstly, and further study proved that the causative agent isolated from ducks is duck Tembusu virus (DTMUV). DTMUV is a single-stranded positive-sense RNA virus classified in the Genus Flavivirus, Family 
Flaviviridae. As with other flaviviruses, mature virions have a diameter of $45-60 \mathrm{~nm}$, its genome is approximately $11 \mathrm{~kb}$ in length. The whole genome is translated into a single polyprotein, which is subsequently processed by viral- and host-encoded proteases into structural and nonstructural proteins (Tang et al. 2012). Three structural proteins (C, $\mathrm{prM} / \mathrm{M}$ and $\mathrm{E}$ ) make up the viral particle and seven nonstructural proteins (NS1, NS2A, NS2B, NS3, NS4A, NS4B and NS5) are required for genome replication and polyprotein processing (Heinz and Stiasny 2012). Similar to the $\mathrm{E}$ proteins in other flaviviruses, the envelope (E) protein of DTMUV is the major surface protein of the virion that mediates binding to the cellular receptor and subsequent fusion event between viral and host membranes (Perera et al. 2008). Also, DTMUV $\mathrm{E}$ is the primary and majority target of neutralizing antibodies. Crystallographic analysis reveals that the E glycoprotein of flaviviruses folds into three distinct structural domains (I, II and III) (Luca et al. 2012). Domain III of flaviviruses E protein (DIII) is the putative receptor-binding domain and is an important target for neutralizing antibodies and in vivo protection (Yu et al. 2013).

However, licensed DTMUV vaccine or drug is currently unavailable. Hence, the development of other technological means against DTMUV is critical for disease control. In viral diseases, for which a specific therapy is not yet available, antibody-based therapy represents a promising alternative strategy. Neutralizing antibodies have been demonstrated to be effective in animal models, such as prophylaxis and as treatments for some flavivirus infections (Morrey et al. 2006, Pierson and Diamond 2008). Also, neutralizing antibodies having strong and specific reactivity to flaviviruses antigens are the most suitable choice for the development of standardized diagnostic tools. In this paper, we described the generation and characterization of a monoclonal antibody, 3E9, specific for the DTMUV E protein. Also, the neutralizing profiles of $\mathrm{mAb} 3 \mathrm{E} 9$ were characterized in vitro and in vivo. The information provided by this research will facilitate the development of diagnostic tools for the specific serological diagnosis of DTMUV infection, and will contribute to the rational design of vaccines by broadering understanding of the antigenic structure of DTMUV E protein.

\section{Materials and Methods}

\section{Cells and viruses}

The myeloma cell line SP2/0 was cultured in Dulbecco's modified Eagle's medium (DMEM, Invitro- gen) in a humidified $5 \% \quad \mathrm{CO}_{2}$ atmosphere at $37^{\circ} \mathrm{C}$. BHK21 cells were cultured in the same condition. All culture media were supplemented with $10 \%$ heat-inactivated fetal bovine serum (GIBCO, Invitrogen), 0.1 $\mathrm{mg} / \mathrm{ml}$ of streptomycin and $100 \mathrm{IU} / \mathrm{ml}$ of penicillin. DTMUV JS804 strain was stored in our laboratory. Also, DTMUV-positive/negative mouse serum were generated and maintained in our laboratory. Female $\mathrm{Balb} / \mathrm{c}$ mice (5-weeks-old) were purchased from Yangzhou University (Jiangsu, China) and raised in special cages with food and water supplied. All animal experiments were carried out in accordance with the regulations and guidelines of animal experimentation outlined by the people's Government of Jiangsu Province (SYXK (Su) 2010-0005).

\section{Expression of recombinant $E$ protein}

The full-length envelope coding sequence was amplified using the primers DTMUV E-EcoRI (5'-CAC A C GAA T T C C G A G A C T T T G T T G A G GGAGTGA-3') and DTMUV E-XhoI (5'- CACACCTCGAGGACATGGATATGGGAACTCTAC-3'). These primers were designed according to the sequence of DTMUV JS804 strain (GenBank No. JF895923) and contained EcoR I and Xho I sites (shown in underline) to facilitate directional cloning into the pET28a $(+)$ expression vector following amplification, agarose gel purification and restriction enzyme digestion. The recombinant plasmid was verified by restriction enzyme digestion and DNA sequencing, then it was transformed into E. coli BL21 cells for expression. After the cells were cultivated to an OD600 (optical density at $600 \mathrm{~nm}$ ) of $0.6-0.8$ in LB media at $37^{\circ} \mathrm{C}, 0.8 \mathrm{mM}$ isopropyl-b-D-1-thiogalactopyranoside (IPTG) was added into the medium to induce the proteins expression for $5 \mathrm{~h}$. Then the cells were harvested by centrifugation and resuspended in phosphate-buffered saline (PBS, pH7.4). Then cell lysates were prepared by sonication and analyzed by SDS-PAGE using $12 \%$ gels to confirm the distribution of the expressed recombinant protein. Finally, the yield of expressed recombinant proteins were analyzed by Western blot.

\section{Preparation and purification of $\mathbf{m A b}$}

Monoclonal antibody (mAb) to the recombinant $\mathrm{E}$ protein was produced using a standard procedure (Lelli et al. 2012). Five-week-old Balb/c female mice were immunized by subcutaneous injection with $70 \mu \mathrm{g}$ of purified recombinant $E$ protein emulsified with an equal volume of Freund's complete adjuvant, then 
followed by two injections at 2 weeks interval with the protein emulsified in the Freund's incomplete adjuvant. Three days after the last boost, the mice were sacrificed and hybridomas were generated following fusion of splenocytes with NS0 myeloma cells and selected cultures were grown following the standard method (Lelli et al. 2012). The fused cells were cultured and selected in RMPI-1640 medium (HAT medium and HT medium). The mAb-producing hybridoma cells were cloned by limiting dilution of the cells three times. Ascites were derived from the mice primed with a $0.5 \mathrm{ml}$ adjuvant and then injected with $2 \times 10^{6}$ hybridoma cells by intraperitoneal injection. E-specific $m A b s$ were identified by immunostaining of BHK21 cells transfected with plasmids expressing the $\mathrm{E}$ protein. The hybridoma-producing $\mathrm{mAb} 3 \mathrm{E} 9$ (IgG1) was cloned twice via limiting dilutions of the cells. mAb 3E9 was purified from ascites by protein A affinity column (GE Healthcare) according to manufacturer's instructions. Briefly, ascites was diluted in $1 \times$ PBS and injected into pre-equilibrated protein A column. The whole antibody was eluted from the column using $25 \mathrm{mM}$ glycine $(\mathrm{pH}$ 2.2) elution buffer according to manufacturer's instructions.

\section{Characterization of mAb 3E9}

mAb 3E9's profiles were characterized by Western blot and Indirect immunofluorescence assay (IFA). BHK21 cells were transiently transfected with recombinant plasmids encoding $\mathrm{E}$ protein or with empty pVAX1 (control) using Lipofectamine 2000 reagent (Invitrogen). $48 \mathrm{~h}$ after transfection, cultured cells were lysed and analyzed by Western blotting using $\mathrm{mAb} 3 \mathrm{E} 9$ as primary antibodies and a HRP-conjugated goat anti-mouse secondary antibody (New England Biolabs, USA). IFA was performed as follows. Briefly, BHK21 cells were transiently transfected with recombinant plasmids encoding $\mathrm{E}$ protein or infected with DTMUV JS804 strain, and then fixed with ice-cold acetone. Cells were incubated with $\mathrm{mAb}$ 3E9. After $60 \mathrm{~min}$ of incubation at $37^{\circ} \mathrm{C}$, cells were washed three times with phosphate-buffered saline (PBS). Cells were then treated with a 200 -fold dilution of FITC-conjugated anti-mouse IgG (KPL) for $30 \mathrm{~min}$ at $37^{\circ} \mathrm{C}$ and rinsed with PBS. After five washes in PBS, positive cells were detected using a fluorescent microscope.

\section{Indirect enzyme-linked immunosorbent assay}

The mAb 3E9 titer of culture supernatant or the ascites was determined by indirect ELISA. Briefly, a 96-well plate was coated with purified recombinant E protein $(2 \mu \mathrm{g} / \mathrm{ml})$ at $4^{\circ} \mathrm{C}$ overnight and blocked with $5 \%$ skimmed milk dissolved in PBS at $37^{\circ} \mathrm{C}$ for $2 \mathrm{~h}$. Then, plates were washed five times with PBST and incubated with different concentrations of 3E9 in triplicate for $1 \mathrm{~h}$ at $37^{\circ} \mathrm{C}$. Plates were washed five times and then incubated with peroxidase-conjugated goat anti-mouse $\operatorname{IgG}$ (1:5000) (New England Biolabs, USA) for $1 \mathrm{~h}$ at $37^{\circ} \mathrm{C}$. Plates were washed five times and then sequentially incubated with TMB substrate (Promega, USA). The reaction was stopped with $2 \mathrm{M} \mathrm{H}_{2} \mathrm{SO}_{4}$ and the OD value of each well was read at $450 \mathrm{~nm}$ using a microplate reader (BioRad 550). The isotype of $\mathrm{mAb} 3 \mathrm{E} 9$ was determined using a Mouse Monoclonal Antibody Isotyping Kit (Promega, USA).

\section{Neutralization assay}

The competence of $\mathrm{mAb} 3 \mathrm{E} 9$ to neutralize virus infectivity was carried out in 96-well microplates. Purified $\mathrm{mAb} 3 \mathrm{E} 9$ was serially diluted fourfold in DMEM, and $100 \mu \mathrm{l}$ aliquots of each dilution were mixed with $100 \mu 1$ 100TCID $_{50}$ DTMUV JS804 strain. The antibody-virus mixtures were incubated for $1 \mathrm{~h}$ at $37^{\circ} \mathrm{C}$ and then transferred to 96-well plates containing $85 \%-95 \%$ confluent monolayers of BHK-21 cells. After incubation for $72-96$ hours at $37^{\circ} \mathrm{C}$ with $5 \%$ $\mathrm{CO}_{2}$, wells were scored for cytopathic effect and neutralizing titers were expressed as the reciprocal of the final $\mathrm{mAb}$ dilution required to neutralize $100 \%$ of the inoculated cultures.

For neutralization assay in vivo, purified $\mathrm{mAb} 3 \mathrm{E} 9$ was serially diluted fourfold in DMEM, and $100 \mu \mathrm{l}$ aliquots of each dilution were mixed with $100 \mu \mathrm{l} 200$ $\mathrm{LD}_{50} / 0.025 \mathrm{ml}$ DTMUV. The antibody-virus mixtures were incubated for $1 \mathrm{~h}$ at $37^{\circ} \mathrm{C}$. Then $0.025 \mathrm{ml}$ $\mathrm{mAb}$-virus mixture was injected intracerebrally into 3-day-old suckling mouse. The control group only received PBS diluent. The animals were monitored daily for clinical signs of infection, including ruffled hair, a hunched back, paralysis, and death, for 10 days. The neutralization activity was calculated by using Reed and Muench's method.

\section{Cell-binding assay}

$50 \mu \mathrm{g} / \mathrm{ml} \mathrm{mAb} 3 \mathrm{E} 9$ or $3 \mathrm{H} 11$ (another monoclonal antibody without neutralizing activity) or BSA was incubated with 500TCID 50 DTMUV JS804 strain for 60 $\min$ at $4^{\circ} \mathrm{C}$. The virus-mAb mixtures were then added to BHK21 cells in 24-well plates for $60 \mathrm{~min}$ on ice. Unbound virus was removed after three washes with PBS. Total viral RNA was extracted from infected 
A

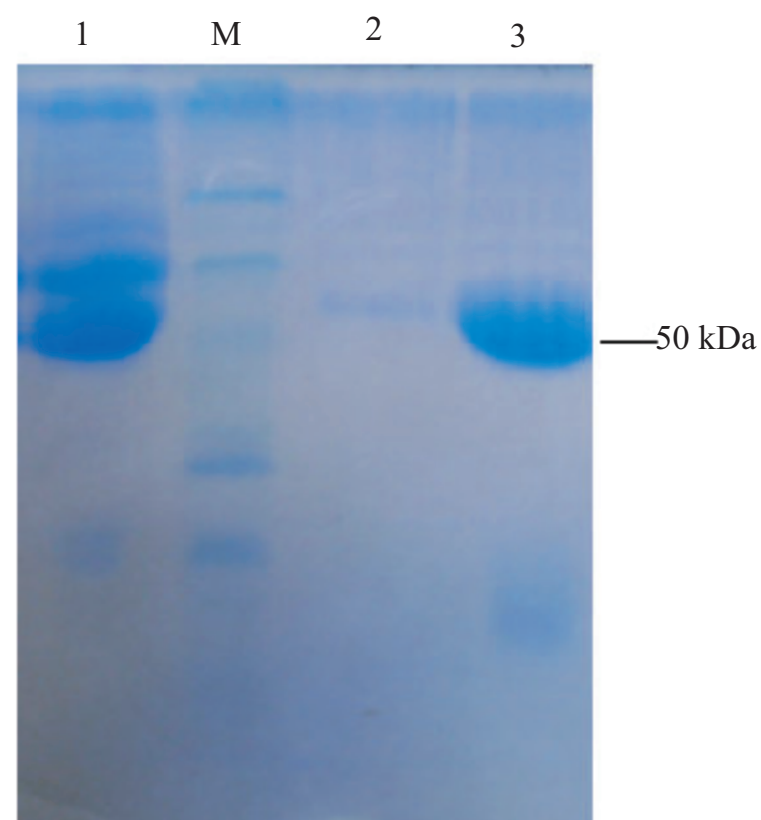

$\mathrm{B}$

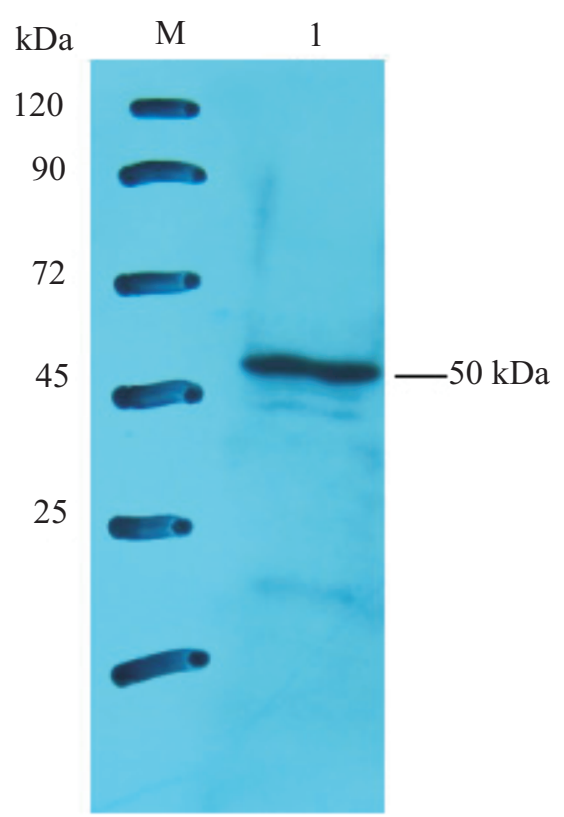

Fig. 1. (A) The expressed and purified recombinant E protein was detected by $12 \%$ SDS-PAGE. Lane M: Protein marker; Lane 1: induced pET28a-E; Lane 2: negative control pET28a; Lane 3: induced and purified recombinant E protein. (B) Western blot analysis of the purified E protein. Lane M: Protein marker; Lane 1: purified E protein.

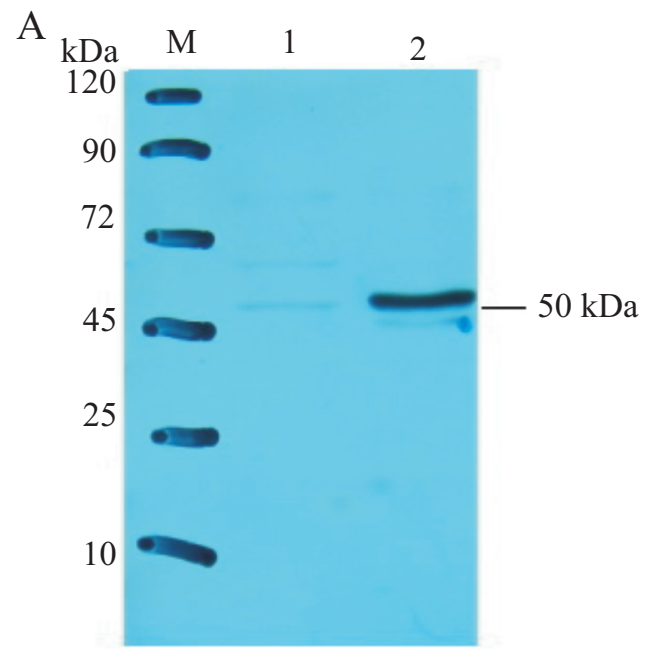

B
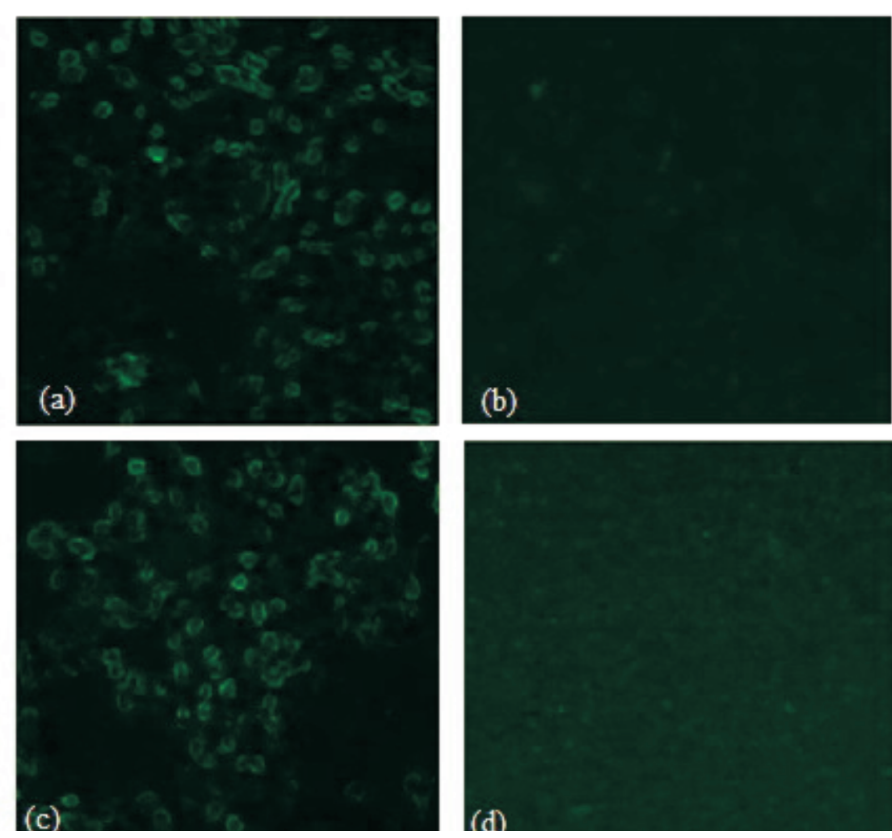

Fig. 2. Characterization of mAb 3E9 in vitro. (A) The specificity of 3E9 for the DTMUV native E protein. BHK21 cells were transiently transfected with the recombinant plasmid encoding the E protein or with a control vector, empty $\mathrm{pVAX} 1.48 \mathrm{~h}$ after transfection, cultured cells were lysed and analyzed by Western Blotting with mAb 3E9. Lane M: Protein marker; Lane 1: empty vector pVAX1; Lane 2: the recombinant plasmid pVAX1-E.; (B) Reactivity of mAb 3E9 with DTMUV native E protein and duck Tembusu virus determined by indirect immunofluorescence analysis. (a) BHK21 cells were transiently transfected with the recombinant plasmid encoding the E protein; (b) BHK21 cells were transiently transfected with the plasmid pVAX1. (c) BHK21 cells were infected with DTMUV. Three to five days after infection, cells were fixed and analyzed by IFA with mAb 3E9. (d) Uninfected BHK-21 cells were run simultaneously as negative controls.

cells using Axygen Total RNA extraction Kit (Axygen Biosciences, China) according to the manufacturer's instructions and viral RNA was quantified by real-time RT-PCR as previously reported (Zheng et al. 2008).

\section{Pre- and post-adsorption inhibition assay}

Neutralization of DTMUV before or after adsorption to BHK21 cells was performed using $1_{00 T_{C I D}}$ of DTMUV and serial dilutions of 3E9 essentially 
as above. In the pre-adsorption assay, the $\mathrm{mAb}$ was firstly incubated with BHK21 cells for $60 \mathrm{~min}$ at $4^{\circ} \mathrm{C}$, then the DTMUV was added and incubated for additional $60 \mathrm{~min}$ at $4^{\circ} \mathrm{C}$. In the post-adsorption assay, DTMUV firstly were added to BHK21 cells for 60 min at $4^{\circ} \mathrm{C}$, then the $\mathrm{mAb} 3 \mathrm{E} 9$ was added and incubated for additional $60 \mathrm{~min}$ at $4^{\circ} \mathrm{C}$.

\section{Results}

\section{Expression and purification of $\mathbf{E}$ protein}

Recombinant DTMUV E protein was successfully expressed in E. coli BL21 (DE3) and purified by Ni-NTA purification system (Merck). The molecular weight of the expressed recombinant $\mathrm{E}$ protein was approximate $50 \mathrm{kDa}$ as determined by $12 \%$ SDS-PAGE, consistent with the molecular weight of the predicted protein (Fig. 1A). The expression of recombinant $\mathrm{E}$ protein was further confirmed by Western blot with mouse anti-serum to DTMUV. The band was in agreement with the predicted size from the prokaryotic expressed protein (Fig. 1B). These results indicated that recombinant $\mathrm{E}$ protein, possessing good reactivity with corresponding anti-serum, was correctly expressed and well purified.

\section{Generation and characterization of mAb 3E9}

Purified E protein was used to immunize $\mathrm{Balb} / \mathrm{c}$ mice. After cell fusion and screening, several hybridoma cell lines were obtained which produced E-specific mAbs. Among them one cell line, 3E9, was selected for its strongest reactivity against recombinant E proten using indirect ELISA, WB (Fig. 2A), and against native $\mathrm{E}$ protein in IFA using DTMUV antigen slides (Fig. 2B). Also, the 3E9 mAb recognized the DTMUV by IFA (Fig. 2B). The subtypes of $\mathrm{mAb}$ 3E9 were determined using the Mouse Monoclonal Antibody Isotyping Kit (Promega, USA) according to the manufacturer's instructions. It was shown that the heavy chain of 3E9 was IgG1 and the light chain was $\kappa$ type. Antibody titers of culture supernatants and the ascites prepared with the hybridoma cell line were measured by indirect ELISA. Antibody titer of the culture supernatants of mAb 3E9 was 1:512, and Antibody titer of the ascite was 1:512,000.

\section{Neutralizing Activity}

This was analyzed for mAb 3E9 using the neutralization assay described above. 3E9 supernatant showed strong neutralization activity up to 1:50 dilu- tion. Since the specific IgG1 concentration in the supernatant was $0.2 \mathrm{mg} / \mathrm{ml}$ it can be extrapolated that 3E9 efficiently neutralized DTMUV at concentrations of at least $4 \mu \mathrm{g} / \mathrm{ml}$. To characterize the neutralizing Activity of $3 \mathrm{E} 9$ in vivo, an existed suckling mouse model was utilized to analyze the protective efficacy of 3E9 against lethal DTMUV infection. The DTMUV infected group developed clinical symptoms and died 4 to 6 days post-infection. mAb 3E9 treatment showed protection against DTMUV in a dose-dependent manner and strong neutralization activity up to 1:15 dilution. Statistical analysis showed that survival rate of 3E9-treated mice were significantly higher than that of PBS-treated group, indicating that $3 \mathrm{E} 9$ confers protection against DTMUV infection in vivo.

\section{Cell-binding assay}

In order to explore the mechanism of 3E9-mediated neutralization of DTMUV further, cell-binding assay was performed with BHK21 cells. Another E-specific mAb (3H11) without neutralizing activity and BSA were used as controls. The results showed that 3E9 could more significantly $(\mathrm{p}<0.01)$ inhibit DTMUV binding than $3 \mathrm{H} 11$, whereas BSA protein failed to inhibit virus binding (Fig. 3). This phenomenon suggested that the activity of 3E9 may involve the blockade of cell attachment.

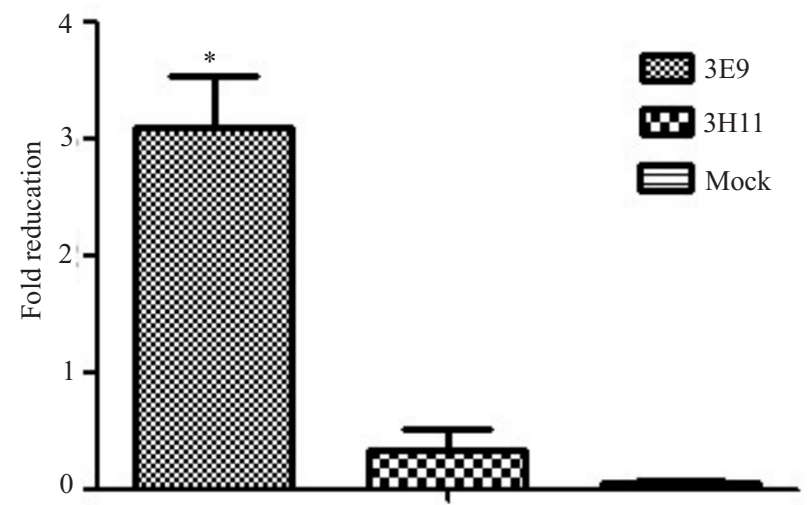

Fig. 3. Mechanism of mAb 3E9-mediated neutralization. Duck Tembusu virus binding to BHK21 cells. The E-specific neutralizing mAb 3E9 blocked cellular attachment significantly more than mAb 3H11 and BSA control. Fold expressions are reported with standard deviations.

\section{Pre- and post-adsorption inhibition assay}

In the pre- and post-adsorption inhibition assay (Fig. 4), 3E9 was incubated with DTMUV before or after its binding to a monolayer of BHK21 cells, also 


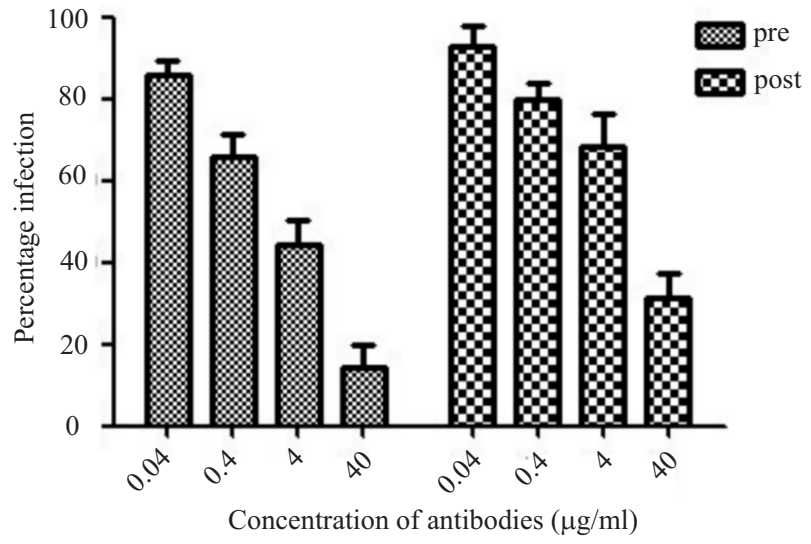

Fig. 4. Pre- and post-adsorption inhibition assays. Pre-binding of DTMUV with 3E9 significantly protected against DTMUV infection (Pre). Infection percentages at the different antibody concentrations are shown with standard deviations.

virus titer was measured as above described. The results showed 3E9 neutralized infection when applied before the binding of the virus. These findings indicated that mAb 3E9-mediated neutralization activity occurs primarily before DTMUV cellular attachment.

\section{Discussion}

In the present study, we generated and characterized a monoclonal antibody (3E9) against DTMUV $\mathrm{E}$ protein. mAb $3 \mathrm{E} 9$ has strong neutralizing activity with duck Tembusu virus in vitro and could protect mice against DTMUV infection in vivo. Furthermore, functional experiments suggested that $\mathrm{mAb} 3 \mathrm{E} 9$ neutralized viral infectivity at a pre-attachment step in the viral life cycle.

E protein is an important structural protein of flaviviruses. By analogy with well-studied flaviviruses, the E protein of DTMUV is the major surface protein of the virion that mediates binding to the cellular receptor and subsequent fusion event between viral and host membranes (Mukhopadhyay et al. 2005). Similar to the $\mathrm{E}$ proteins in other flaviviruses, DTMUV E is the primary target of neutralizing antibodies (Liu et al. 2012). The functional and immunobiological characteristics of the $\mathrm{E}$ protein make it a good candidate for development of a prophylactic vaccine (Azevedo et al. 2011, Coller et al. 2011). In our study, we injected mice with recombinant $\mathrm{E}$ protein and isolated a $\mathrm{mAb}$ that recognized a protein band of around 50 $\mathrm{kDa}$ in Western blot, corresponding to $\mathrm{E}$ protein. By IFA, we found the mAb $3 \mathrm{E} 9$ could recognize the E protein expressed in eukaryotic cells or whole duck Tembusu viron, indicating that the epitope recognized by the $\mathrm{mAb} 3 \mathrm{E} 9$ is exposed on the $\mathrm{E}$ protein surface. In our study, we also found that mAb 3E9 could sig- nificantly inhibit cellular attachment of DTMUV to BHK21 cells, and it inhibited infection when added before virus binding, suggesting that $\mathrm{mAb} 3 \mathrm{E} 9$ neutralizes viral infectivity at a pre-attachment step in the viral life cycle. Generally, antibodies have the potential to neutralize the infectivity of flaviviruses by interfering with several steps of the virus entry pathway, such as attachment, internalization and fusion, or depending on the locations of their binding sites (Oliphant and Diamond 2007). Our finding is similar to Oliphant's research, which reported mAb E53 of West Nile virus inhibited infection primarily by blocking viral attachment to some cells (Oliphant et al. 2006).

DTMUV is an important poultry pathogen that causes substantial egg production drop in infected ducks (Cao et al. 2011, Su et al. 2011, Ti et al. 2015). DTMUV-induced egg production drops also observed in other domestic birds, including hens and geese, caused lots of economic losses to the poultry industry in China. But up to now, specific antiviral therapy or vaccines are absent for DTMUV. Vaccination is efficient and fast to DTMUV control, but no TMUV successful commercial vaccine has been developed and used in the field. Several inactivated TMUV vaccine candidates have been developed and used in some areas but these inactivated vaccines might not elicit a robust neutralizing antibody response (Sun et al. 2014). Therefore, the need for development of alternative ways against TMUV appeared to gain high priority. Monoclonal antibodies have become a powerful tool to study protein structure and have been widely used to diagnose and treat a variety of infectious agents (Denisova et al. 2008, Shen et al. 2009, Sukupolvi-Petty et al. 2010). The mAb 3E9 presented herein may advantageously be used to treat or prevent DTMUV infection in mice, for this reason, mAb 3E9 may further be developed as a therapeutic antibody to treat DTMUV in ducks after further investigation.

\section{Acknowledgements}

This work was supported by National Natural Science Foundation of China (No. 31172345; No. 31502101), the Jiangsu Natural Sciences Foundation (BK20130710; BK20160064), Jiangsu Provincial Agricultural Science and Technology Innovation Foundation [No. cx (12) 5048], the China Postdoctoral Science Foundation Grant [2016M590430].

\section{References}

Azevedo AS, Yamamura AM, Freire MS, Trindade GF, Bonaldo M, Galler R, Alves AM (2011) DNA vaccines 
against Dengue virus type 2 based on truncate envelope protein or tts domain III. PLoS ONE 6: e20528.

Cao Z, Zhang C, Liu Y, Liu Y, Ye W, Han J, Ma G, Zhang D, Xu F, Gao X, Tang Y, Shi S, Wan C, Zhang C, He B, Yang M, Lu X, Huang Y, Diao Y, Ma X, Zhang $D$ (2011) Tembusu virus in ducks, China. Emerg Infect Dis 17: 1873-1875.

Coller BA, Clements DE, Bett AJ, Sagar SL, Ter Meulen JH (2011) The development of recombinant subunit envelope-based vaccines to protect against Dengue virus induced disease. Vaccine 29: 7267-7275.

Denisova GF, Denisov DA, Yeung J, Loeb MB, Diamond MS, Bramson JL (2008) A novel computer algorithm improves antibody epitope prediction using affinity-selected mimotopes: a case study using monoclonal antibodies against the West Nile virus E protein. Mol Immunol 46: 125-134.

Heinz FX, Stiasny K (2012) Flaviviruses and their antigenic structure. J Clin Virol 55: 289-295.

Lelli D, Moreno A, Brocchi E, Sozzi E, Capucci L, Canelli E, Barbieri I, Zeller H, Cordioli P (2012) West Nile virus: characterization and diagnostic applications of monoclonal antibodies. Virol J 9: 81.

Liu P, Lu H, Li S, Moureau G, Deng YQ, Wang Y, Zhang L, Jiang T, de Lamballerie X, Qin CF, Gould EA, Su J, Gao GF (2012) Genomic and antigenic characterization of the newly emerging Chinese duck egg-drop syndrome flavivirus: genomic comparison with Tembusu and Sitiawan viruses. J Gen Virol 93: 2158-2170.

Luca VC, AbiMansour J, Nelson CA, Fremont DH (2012) Crystal structure of the Japanese encephalitis virus envelope protein. J Virol 86: 2337-2346.

Morrey JD, Siddharthan V, Olsen AL, Roper GY, Wang H, Baldwin TJ, Koenig S, Johnson S, Nordstrom JL, Diamond MS (2006) Humanized monoclonal antibody against West Nile virus envelope protein administered after neuronal infection protects against lethal encephalitis in hamsters. J Infect Dis 194: 1300-1308.

Mukhopadhyay S, Kuhn RJ, Rossmann MG (2005) A structural perspective of the flavivirus life cycle. Nat Rev Microbiol 3: 13-22.

Oliphant T, Diamond MS (2007) The molecular basis of antibody-mediated neutralization of West Nile virus. Expert Opin Biol Th 7: 885-892.

Oliphant T, Nybakken GE, Engle M, Xu Q, Nelson CA, Sukupolvi-Petty S, Marri A, Lachmi BE, Olshevsky U, Fremont DH, Pierson TC, Diamond MS (2006) Antibody recognition and neutralization determinants on domains I and II of West Nile Virus envelope protein. J Virol 80: 12149-12159.
Perera R, Khaliq M, Kuhn RJ (2008) Closing the door on flaviviruses: entry as a target for antiviral drug design. Antivir Res 80: 11-22.

Pierson TC, Diamond MS (2008) Molecular mechanisms of antibody-mediated neutralization of flavivirus infection. Expert Rev Mol Med 10: e12.

Shen X, Parks RJ, Montefiori DC, Kirchherr JL, Keele BF, Decker JM, Blattner WA, Gao F, Weinhold KJ, Hicks CB, Greenberg ML, Hahn BH, Shaw GM, Haynes BF, Tomaras GD (2009) In vivo gp41 antibodies targeting the 2F5 monoclonal antibody epitope mediate human immunodeficiency virus type 1 neutralization Breadth. J Virol 83: 3617-3625.

Su J, Li S, Hu X, Yu X, Wang Y, Liu P, Lu X, Zhang G, Hu X, Liu D, Li X, Su W, Lu H, Mok NS, Wang P, Wang M, Tian K, Gao GF (2011) Duck egg-drop syndrome caused by BYD virus, a new Tembusu-related flavivirus. PLoS One, 6: e18106.

Sukupolvi-Petty S, Austin SK, Engle M, Brien DJ, Dowd AK, Williams LK, Johnson S, Rico-Hesse R, Harris E, Pierson CT, Fremont HD, Diamond SM (2010) Structure and function analysis of therapeutic monoclonal antibodies against Dengue Virus type 2. J Virol 84: 9227-9239.

Sun X, Diao Y, Wang J, Liu X, Lu A, Zhang L, Ge P, Hao $D$ (2014) Tembusu virus infection in Cherry Valley ducks: the effect of age at infection. Vet Microbiol 168: $16-24$.

Sun L, Li Y, Zhang Y, Han Z, Xu Y, Kong X, Liu S (2014) Adaptation and Attenuation of Duck Tembusu Virus Strain Du/CH/LSD/110128 following Serial Passage in Chicken Embryos. Clin Vaccine Immunol 21: 1046-1053.

Tang Y, Diao Y, Gao X, Yu C, Chen L, Zhang D (2012) Analysis of the complete genome of Tembusu virus, a flavivirus isolated from ducks in China. Transbound Emerg Dis 59: 336-343.

Ti J, Zhang L, Li Z, Zhao D, Zhang Y, Li F, Diao Y (2015) Effect of age and inoculation route on the infection of duck Tembusu virus in Goslings. Vet Microbiol 181: 190-197.

Yu K, Sheng ZZ, Huang B, Ma X, Li Y, Yuan X, Qin Z, Wang D, Chakravarty S, Li F, Song M, Sun H (2013) Structural, antigenic, and evolutionary characterizations of the envelope protein of newly emerging duck Tembusu virus. PLoS One, 8: e71319.

Zheng X, Hong L, Shi L, Guo J, Sun Z, Zhou J (2008) Proteomics Analysis of Host Cells Infected with Infectious Bursal Disease Virus. Mol Cell Proteomics 7: 612-625. 